УДК $532.711+66.081 .6$

\title{
Modelling of Electrochemically Switchable Ion Transport in Nanoporous Membranes with Conductive Surface
}

\author{
Ilya I. Ryzhkov* \\ Anton S. Vyatkin ${ }^{\dagger}$ \\ Institute of Computational Modelling SB RAS \\ Akademgorodok, 50-44, Krasnoyarsk, 660036 \\ Siberian Federal University \\ Svobodny, 79, Krasnoyarsk, 660041 \\ Russia \\ Maria I. Medvedeva $a^{\ddagger}$ \\ Siberian Federal University \\ Svobodny, 79, Krasnoyarsk, 660041 \\ Russia
}

Received 25.03.2019, received in revised form 30.05.2019, accepted 02.07.2019

The impact of potential applied to the conductive surface of nanoporous membrane on the membrane potential at zero current is investigated theoretically on the basis of two-dimensional Space-charge model. The membrane separates two reservoirs with different salt concentrations. It is shown that the variation of applied potential from negative to positive values results in the continuous change of membrane selectivity from cation to anion. For equal ion diffusion coefficients, the dependence of membrane potential on the applied potential is an odd function, while for different ion diffusion coefficients it is shifted along the applied potential axis due to contribution of diffusion potential enhanced by the induced charge effect. The decrease of pore radius results in the increase of ionic selectivity and steep transition between cationselective and anion-selective states when the applied potential is changing.

Keywords: nanoporous membrane, electric double layer, electrolyte transport, membrane potential, Spacecharge model.

DOI: 10.17516/1997-1397-2019-12-5-579-589.

\section{Introduction}

Over the last two decades, the development of nanopores and nanochannels with controlled ion transport has attracted a lot of research attention. The applications of such structures include separation and purification processes [1], water treatment [2], chemical sensors [3], and synthetic analogues of biological ion channels and pumps [4]. Tunable ion transport can be realized via the interaction of pore geometry and surface physicochemical properties with external stimuli, such as electric field, radiation, temperature, solution $\mathrm{pH}$, etc. [5,6].

The use of electric field for switching the ionic selectivity of a nanoporous membrane was first demonstrated in [7]. The membrane was prepared by electroless plating of gold on the pore walls

\footnotetext{
*rii@icm.krasn.ru

$\dagger_{\mathrm{v}}$ _anton_s@icm.krasn.ru

${ }^{\ddagger}$ mimedvedeva@rambler.ru

(c) Siberian Federal University. All rights reserved
} 
of track-etched polymeric support. It was shown that its selectivity can be continuously and reversibly switched from cation to anion by applying the prescribed potential to the conductive membrane surface [8]. The authors measured the membrane potential at zero current, which results from the Donnan equilibrium between diffusion and electric forces at membrane/solution interfaces and diffusion potential caused by different ion mobilities. The membrane transport experiments in [9] revealed that that the fluxes of anionic and cationic permeate species change as a function of applied potential. The modulation of membrane potential by the external electric field in solid-state channels was also demonstrated in [10], while a significant increase of ionic conductivity in nanopores and nanochannels due to application of a prescribed potential to their conductive surface was reported in [11-13].

Recently, a new type of highly porous ceramic membranes with a conductive coating was suggested $[14,15]$. The membranes were prepared by vacuum filtration of a colloidal solution of Nafen alumina fibers with a diameter of about $10 \mathrm{~nm}$ through a porous substrate and subsequent drying and annealing. The deposition of a thin carbon layer on the nanofibers by chemical vapor deposition (CVD) provided the formation of an electrically conductive membrane surface. The possibility of controlling the membrane potential and ionic conductivity by application of external potential was demonstrated [16]. A new mechanism of membrane potential generation in conductive pores was discovered in [17]. The electric field caused by diffusion of ions with different mobilities induces a surface charge on a conductive pore wall, which results in charge separation inside the nanopore. The corresponding Donnan potentials appear at the pore entrance and exit leading to a dramatic enhancement of membrane potential in comparison with uncharged dielectric membrane. This effect was described theoretically and confirmed experimentally $[18,19]$. Note that the induced-charge electrokinetic phenomena are actively investigated nowadays due to potential applications in microfluidic pumping and mixing, particle manipulation, and capacitive deionization [20,21].

The theory of ion transport in electrochemically switchable conductive membranes based on a number of restrictive assumptions showed only satisfactory agreement with the experimental data [22]. It was shown in [23] that a qualitative similarity between predicted and measured membrane potential can be obtained with one-dimensional transport model by assuming that the applied voltage gives rise to a fixed charge proportional to it. The two-dimensional SpaceCharge model for cylindrical nanopores with constant surface charge density [30,31] was first extended to the case of constant surface potential in [17] and further applied to description of conductive nanopores in $[18,19]$. The latter studies were focused on the situation when the nanopore charge is induced only by the electric field resulting from different mobilities of ions diffusing through membrane.

In this work, we theoretically investigate the case when the membrane ionic selectivity is switched by the external potential applied to the conductive nanopore surface. The dependence of membrane potential at zero current on the applied voltage, electrolyte type, and pore size is discussed and analyzed.

\section{Mathematical model}

Let us consider a porous membrane separating two reservoirs denoted by $L$ (left) and $R$ (right). The reservoirs contain aqueous solutions of the same monovalent and symmetric (1:1) electrolyte with concentrations $C_{L}$ and $C_{R}$, respectively $\left(C_{L}>C_{R}\right)$. The electrolyte diffuses from the reservoir with a higher concentration to that with a lower concentration. The pressures 
in the reservoirs are equal, and there is no electric current between them. The potential difference between reservoirs, which develops due to ions transport through the pore, is denoted by $\Delta \Phi=$ $=\Phi_{R}-\Phi_{L}$. The magnitude and sign of $\Delta \Phi$ characterizes the ionic selectivity of membrane. The case $\Delta \Phi>0$ corresponds to the cation selective membrane, while $\Delta \Phi<0$ is related to anion selective membrane (strictly speaking, this is valid when the ions have the same diffusion coefficients; otherwise, the diffusion potential can contribute the total membrane potential) [18]. The membrane surface is assumed conductive, and its potential is denoted by $\Phi_{s}$. The described configuration exactly corresponds to the one considered in earlier experimental studies $[7,8,16]$. In what follows, we will also consider a membrane with the constant surface charge density $\Sigma_{s}$ and compare the results with those for constant surface potential.

A membrane is modelled as an array of cylindrical pores of length $L_{p}$ and radius $R_{p}$. The cylindrical coordinates $R$ in radial and $Z$ in axial directions are introduced in a single pore (Fig. 1). We employ the continuum model based on two-dimensional Navier-Stokes, NernstPlanck, and Poisson (NS-PNP) equations. For liquid electrolytes, the continuum approach is applicable for nanopores with the diameter of a few nanometers and larger [24]. Alternative methods particularly suited for small pores $(<2 \mathrm{~nm})$ are the molecular dynamics simulations $[25,26]$ and Monte-Carlo simulations [27]. However, it is often not easy to compare the corresponding results with continuum modelling since simulated systems are usually limited to tens of nanometers, time scales of nanoseconds, and extremely strong external fields (pressure or electrical potential gradients) [28]. Such simulations are also very expensive computationally. The limitations of molecular dynamics can be overcome by coarse-grained simulation techniques such as dissipative particle dynamics [29].

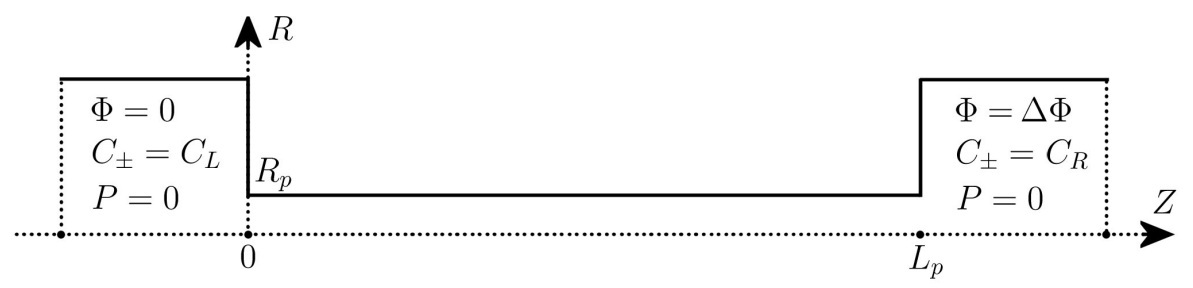

Fig. 1. The geometry of a single cylindrical pore

In the frame of continuum approach, the transport of electrolyte through the pore is characterized by the solution velocity $\boldsymbol{U}=(U, V)$, pressure $P$, cation $C_{+}$and anion $C_{-}$concentrations $\left(\mathrm{mol} / \mathrm{m}^{3}\right)$, and electric potential $\Phi$. The derivation of Space-Charge model from NS-PNP equations and its extension to conductive pore walls are described in details in $[18,19,31]$. Thus, here we will only present the resulting equations and describe the computational procedure.

The ions in the pore are transported by convection, diffusion, and migration in electric field, so their fluxes are written as

$$
\boldsymbol{J}_{ \pm}=C_{ \pm} \boldsymbol{U}-D_{ \pm} \nabla C_{ \pm} \mp \frac{D_{ \pm} F}{R_{g} T} C_{ \pm} \nabla \Phi,
$$

where $D_{ \pm}$are the ion diffusion coefficients, $R_{g}$ is the ideal gas constant, $T$ is the temperature, and $F$ is the Faraday constant. Let us introduce dimensionless variables by

$$
\begin{gathered}
R=R_{p} r, \quad Z=L_{p} z, \quad \boldsymbol{U}=\frac{D_{-}}{L_{p}} \boldsymbol{u}, \quad P=C_{0} R_{g} T p, \quad C_{ \pm}=C_{0} c_{ \pm}, \\
\Phi=\frac{R_{g} T}{F} \varphi, \quad \boldsymbol{J}_{ \pm}=\frac{D_{-} C_{0}}{L_{p}} \boldsymbol{j}_{ \pm}, \quad \sigma_{s}=\frac{\Sigma_{s}}{\varepsilon \varepsilon_{0} R_{g} T / F R_{p}}, \quad \varphi_{s}=\frac{\Phi_{s}}{R_{g} T / F} . \\
-581-
\end{gathered}
$$


Here $\boldsymbol{u}=(u, v)$ and $C_{0}$ is the reference concentration taken as $C_{0}=1 \mathrm{~mol} / \mathrm{m}^{3}$.

In what follows, we will need the quantities averaged over the pore cross-section. The dimensional average axial velocity is defined by

$$
\bar{V}=\frac{2}{R_{p}^{2}} \int_{0}^{R_{p}} V R d R
$$

The average pressure $\bar{P}$, ion concentrations $\bar{C}_{ \pm}$, potential $\bar{\Phi}$, axial ion fluxes $\bar{J}_{ \pm}$, total axial ion flux $\bar{J}=\bar{J}_{+}+\bar{J}_{-}$, and axial ion current $\bar{I}=\bar{J}_{+}-\bar{J}_{-}$are introduced in the same way. The corresponding dimensionless quantities are $\bar{v}, \bar{p}, \bar{c}_{ \pm}, \bar{\varphi}, \bar{j}_{ \pm}, \bar{j}=\bar{j}_{+}+\bar{j}_{-}$, and $\bar{i}=\bar{j}_{+}-\bar{j}_{-}$.

The Space-Charge model is a valid approximation for large aspect ratio pores $\left(L_{p} / R_{p} \gg 1\right)$. The dimensionless potential $\varphi$, ion concentrations $c_{ \pm}$, and pressure $p$ are represented as [31]

$$
\begin{gathered}
\varphi(r, z)=\phi_{v}(z)+\psi(r, z), \quad c_{ \pm}(r, z)=c_{v}(z) \exp (\mp \psi(r, z)), \\
p(r, z)=p_{v}(z)+2 c_{v}(z) \cosh (\psi(r, z)) .
\end{gathered}
$$

Here the ion concentrations satisfy the Boltzmann distribution.

The function $\psi$ satisfies the Poisson equation with boundary condition of axial symmetry

$$
\begin{gathered}
\frac{1}{r} \frac{\partial}{\partial r}\left(r \frac{\partial \psi(r, z)}{\partial r}\right)=\frac{c_{v}(z)}{\lambda^{2}} \sinh \psi(r, z), \\
\frac{\partial \psi}{\partial r}(0, z)=0 .
\end{gathered}
$$

Here $\lambda=\sqrt{\varepsilon \varepsilon_{0} R_{g} T / 2 F^{2} C_{0}} / R_{p}$ is the dimensionless Debye length. The constant surface charge density is imposed by

$$
\frac{\partial \psi}{\partial r}(1, z)=\sigma_{s}
$$

while for the constant surface potential one should write

$$
\psi(1, z)=\varphi_{s}-\phi_{v}(z)
$$

The virtual pressure $p_{v}$, concentration $c_{v}$, and potential $\phi_{v}$ satisfy the system of ODE in the form of phenomenological flux-force formalism:

$$
\left(\frac{d p_{v}}{d z}, \frac{1}{c_{v}} \frac{d c_{v}}{d z}, \frac{d \phi_{v}}{d z}\right)^{T}=L(\bar{v}, \bar{j}, \bar{i})^{T} .
$$

Here $L=-\mathcal{L}^{-1}$ is the symmetric $3 \times 3$ matrix. The coefficients of matrix $\mathcal{L}=\left\{\mathcal{L}_{i j}(z)\right\}$ depend on the function $\psi(r, z)$ and virtual concentration $c_{v}(z)$ according to [18]

$$
\begin{gathered}
\mathcal{L}_{11}=\frac{1}{8 \alpha}, \quad \mathcal{L}_{22}=2 c_{v} \int_{0}^{1} r(D \exp (\psi(r))+\exp (-\psi(r))) d r- \\
-\frac{16 c_{v}^{2}}{\alpha} \int_{0}^{1}\left[r \cosh (\psi(r)) \ln r\left(\frac{r^{2}}{2} \cosh (\psi(r))-\frac{\lambda^{2}}{4 c_{v}}\left(r \frac{\partial \psi(r)}{\partial r}\right)^{2}\right)\right] d r \\
\mathcal{L}_{33}=-\frac{8 c_{v}}{\alpha} \int_{0}^{1} r\left[\sinh (\psi(r)) \lambda^{2}\left(\psi(r)-\psi_{s}\right)-\frac{\alpha}{4}(D \exp (\psi(r))+\exp (-\psi(r)))\right] d r \\
\mathcal{L}_{12}=\mathcal{L}_{21}=\frac{c_{v}}{\alpha} \int_{0}^{1}\left(r-r^{3}\right) \cosh (\psi(r)) d r, \quad \mathcal{L}_{13}=\mathcal{L}_{31}=\frac{4}{\alpha} \int_{0}^{1} r \lambda^{2}\left(\psi(r)-\psi_{s}\right) d r
\end{gathered}
$$




$$
\mathcal{L}_{23}=\mathcal{L}_{32}=\frac{8 c_{v}}{\alpha} \int_{0}^{1} r\left[\cosh (\psi(r)) \lambda^{2}\left(\psi(r)-\psi_{s}\right)-\frac{\alpha}{4}(D \exp (\psi(r))-\exp (-\psi(r)))\right] d r,
$$

where $D=D_{+} / D_{-}, \alpha=\mu D_{-}\left(C_{0} R_{g} T R_{p}^{2}\right)^{-1}, \psi_{s}=\psi(1, z)$, and $\mu$ is the solution dynamic viscosity. The dependence of $\psi$ and $c_{v}$ on $z$ is not explicitly stated in the above formulas.

The boundary conditions correspond to equal (zero) pressures and constant concentrations in the reservoirs, and zero potential at the left reservoir taken as a reference. By putting $\psi(r, z)=0$ in (2), one arrives at

$$
\begin{aligned}
& z=0: \quad p_{v}=-2 c_{L}, \quad c_{v}=c_{L}, \quad \phi_{v}=0, \\
& z=1: \quad p_{v}=-2 c_{R}, \quad c_{v}=c_{R}, \quad \phi_{v}=\Delta \varphi .
\end{aligned}
$$

Here $\Delta \varphi$ is the dimensionless potential difference between the reservoirs (membrane potential).

The membrane potential is measured at zero current $(\bar{i}=0)$. Integration of equations $(7)$ over the pore length with the help of boundary conditions (9) gives [18]

$$
\begin{gathered}
\int_{c_{L}}^{c_{R}} \frac{L_{11} \bar{f}+L_{12}}{c_{v}\left(L_{12} \bar{f}+L_{22}\right)} d c_{v}+2\left(c_{R}-c_{L}\right)=0, \\
\bar{j}=\int_{c_{L}}^{c_{R}} \frac{d c_{v}}{c_{v}\left(L_{12} \bar{f}+L_{22}\right)}, \\
\phi_{v}\left(c_{v}\right)=\int_{c_{L}}^{c_{v}} \frac{L_{13} \bar{f}+L_{23}}{c_{v}\left(L_{12} \bar{f}+L_{22}\right)} d c_{v},
\end{gathered}
$$

where $\bar{f}=\bar{v} / \bar{j}$ is the fluxes ratio. It follows from (3), (6), (8), and (12) that one can write $\psi=\psi\left(r, c_{v}\right), \phi_{v}=\phi\left(c_{v}\right), L_{i j}=L_{i j}\left(c_{v}\right)$.

The calculation is performed as follows. For a dielectric pore with constant surface charge density $\sigma_{s}$, problem (3)-(5) is solved numerically for a set of successive values $c_{v}=c_{v k}, k=0, \ldots, n$, where $c_{v 0}=c_{L}, c_{v n}=c_{R}$. Then the ratio of fluxes $\bar{f}=\bar{v} / \bar{j}$ is found numerically from (10), and the ion flux $\bar{j}$ is obtained from (11). The potential difference between reservoirs $\Delta \varphi=\phi_{v}\left(c_{R}\right)$ is determined from (12), while the virtual variables are found by integration of (7), (9).

For a conductive pore with constant potential $\varphi_{s}$, problem (3), (4), (6) is solved for each $c_{v k}$ at fixed $\varphi_{s}$ and $\bar{j}$. Note that $\phi_{v}\left(c_{v 0}\right)=\phi_{v}\left(c_{L}\right)=0$. The value $\phi_{v}\left(c_{v k}\right)$ is found iteratively starting from $\phi_{v}\left(c_{v, k-1}\right)$ and repeating the solution of (3), (4), (6) followed by application of (12). The calculation is performed iteratively to find the fluxes ratio $\bar{f}$ from (10). Then $\bar{j}$ is calculated from (11) and virtual variables are obtained by integration of (7), (9).

The integration of Poisson equation (3) is performed by reducing it to two first-order ODE and applying the Runge-Kutta-Merson method of 5th order starting from $r=\epsilon$ to $r=1$, where $\epsilon$ is close to zero. Here an additional boundary condition $\psi(\epsilon, x)=\psi_{0}$ is required. The value $\psi_{0}$ is determined by the shooting method in order to satisfy boundary condition (5) or (6) at fixed $z$. The initial approximation for it can be found from analytical solution derived in [32].

\section{Results and discussion}

The dependence of membrane potential on the logarithm of concentrations ratio for different surface charge densities is shown in Fig. 2 (a) for an aqueous electrolyte with equal ion diffusion coefficients. When the surface charge density changes from negative to positive values, the membrane potential varies from positive to negative values. It indicates the continuous change 
(a)

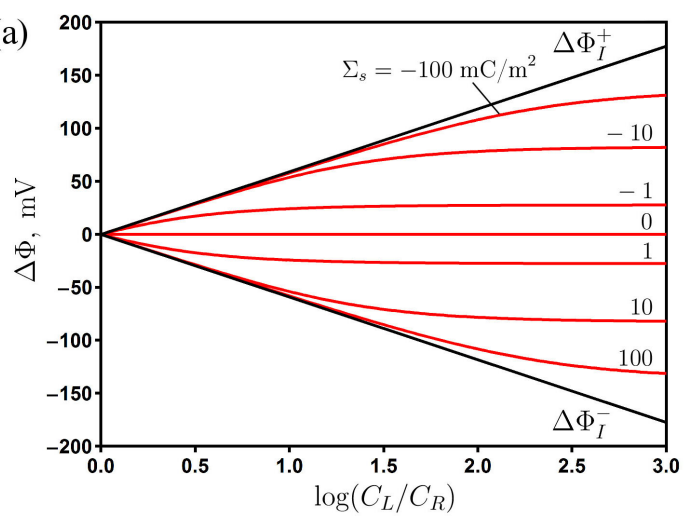

(c)

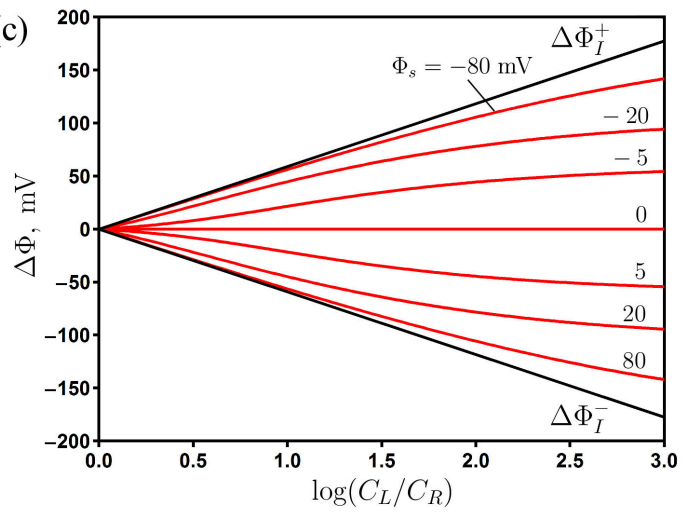

(b)

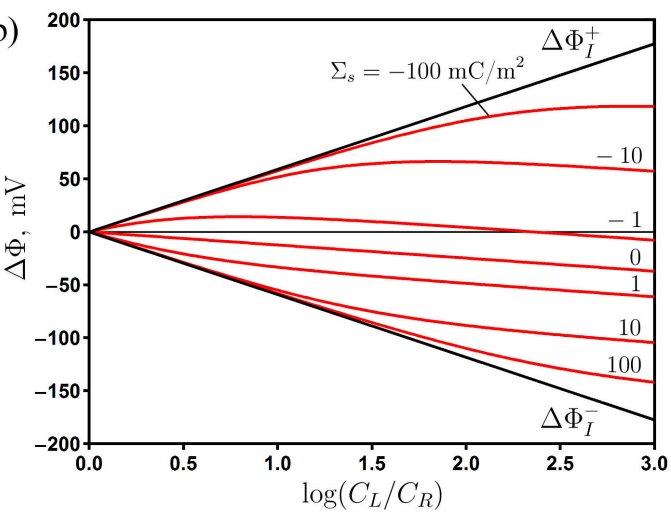

(d)

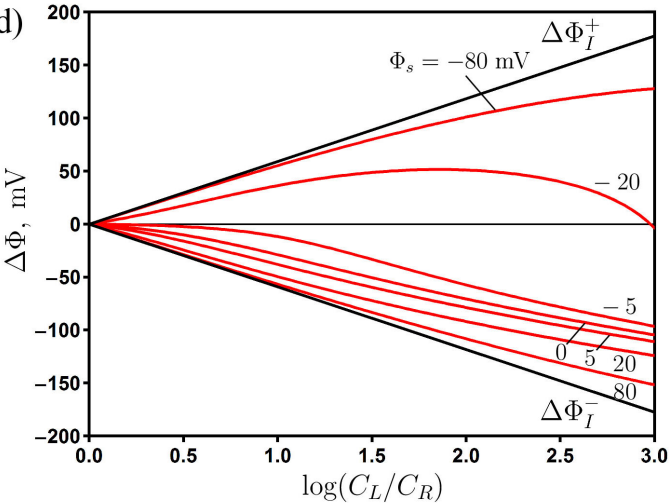

Fig. 2. The dependence of membrane potential on the logarithm of concentrations ratio for different surface charge densities $(\mathrm{a}, \mathrm{b})$ and different surface potentials $(\mathrm{c}, \mathrm{d})$. The electrolyte with equal ion diffusion coefficients $D_{+} / D_{-}=1(\mathrm{a}, \mathrm{c})$ and aqueous $\mathrm{NaCl}$ solution with $D_{+} / D_{-}=0.65(\mathrm{~b}, \mathrm{~d})$. $R_{p}=8 \mathrm{~nm}, L_{p}=4000 \mathrm{~nm}$

of nanopore selectivity from cation to anion. The black lines corresponding to ideal cation (anion) selectivity are described by the formulas [18]

$$
\Delta \Phi_{I}^{ \pm}= \pm \frac{R_{g} T}{F} \ln \frac{C_{L}}{C_{R}} .
$$

The uncharged nanopore $\left(\Sigma_{s}=0 \mathrm{mC} / \mathrm{m}^{2}\right)$ is not selective. The membrane potential curves corresponding to positive and negative values of surface charge density are symmetric with respect to the line $\Sigma_{s}=0$, which coincides with the horizontal axis. The membrane potential for aqueous $\mathrm{NaCl}$ electrolyte is presented in Fig. 2 (b). In this case, the symmetry of the curves is lost due to a small negative contribution of diffusion potential to the total membrane potential value. Note that $D_{+} / D_{-}=0.65$ for $\mathrm{NaCl}$ electrolyte.

When the surface potential is varied in an electrolyte with equal ion diffusion coefficients (Fig. 2 (c)), the situation is similar to the case of surface charge density variation (Fig. 2 (a)). However, for aqueous $\mathrm{NaCl}$ electrolyte, the situation becomes drastically different, see Fig. 2 (d). In this case, the diffusion potential greatly enhances and significantly affects the membrane potential curves. This effect can be explained by considering an example of initially uncharged pore with electrically conductive walls $[18,19]$. The profiles of potential and ion concentrations 

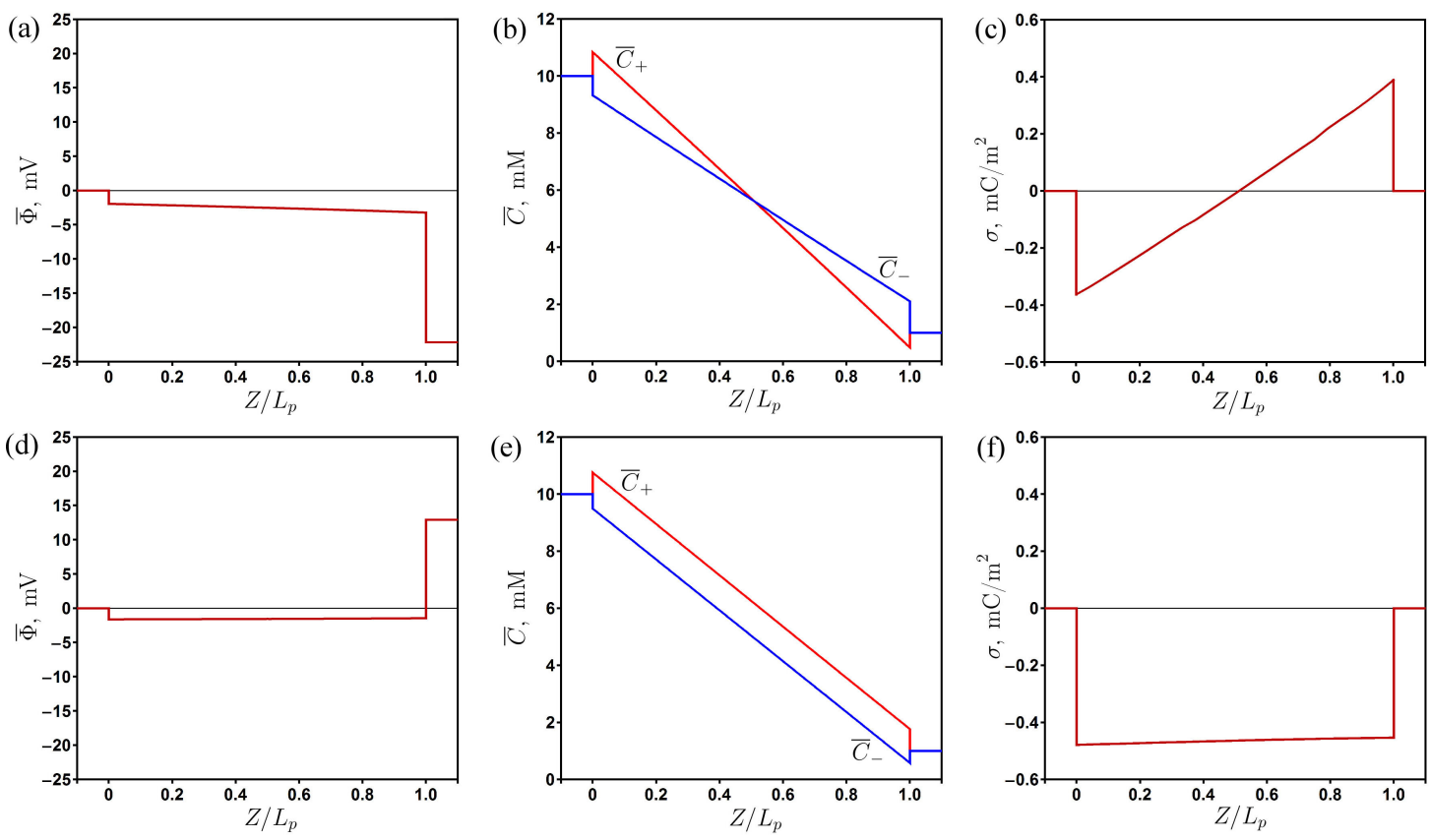

Fig. 3. The cross-sectionally averaged profiles of potential (a,d), ion concentrations (b,e), and surface charge $(\mathrm{c}, \mathrm{f})$ for aqueous $\mathrm{NaCl}$ solution with $D_{+} / D_{-}=0.65(\mathrm{a}, \mathrm{b}, \mathrm{c})$ and electrolyte with equal ion diffusion coefficients $D_{+} / D_{-}=1$ (d,e,f). $\Phi_{s}=-2.7 \mathrm{mV}, R_{p}=8 \mathrm{~nm}, L_{p}=4000 \mathrm{~nm}$

averaged over the pore cross-section as well as surface charge density for this case are shown in Fig. 3. The electric field arising from diffusion of ions with different mobilities induces a surface charge on the wall (i.e. redistributes the electrons there), see Fig. 3 (c). It results in charge separation inside the nanopore (Fig. 3 (b)). The corresponding Donnan potentials appear at the pore entrance and exit contributing to the total negative value of membrane potential (Fig. 3 (a)). The surface potential in this case is set to $\Phi_{s}=-2.7 \mathrm{mV}$, which corresponds to zero total charge of the pore wall. When the same surface potential is applied in the case of equal ion diffusion coefficients, the longitudinal electric field does not arise, and surface charge density stays almost constant and negative, see Fig. 3 (f). A slight excess of cations inside the pore is observed (Fig. 3 (e)), and the value of membrane potential is positive as it should be for cation selective nanopore, Fig. 3 (d). The suggested reasoning explains the shift of membrane potential curves in negative direction in Fig. 2 (d). However, when the applied surface potential is rather large $(\Phi= \pm 80 \mathrm{mV})$, the curves are not significantly affected by the presence of ions with different diffusion coefficients, compare Figs. 2 (c) and (d).

The dependence of membrane potential on the potential applied to the nanopore surface for different electrolytes is shown in Fig. 4 (a). The concentrations in the reservoirs are fixed at $C_{L}=100 \mathrm{mM}, C_{R}=0.1 \mathrm{mM}$. The change of surface potential from negative to positive values leads to membrane potential variation from positive to negative values, which indicates the continuous change of selectivity from cation to anion. When the ion diffusion coefficients are equal $\left(D_{+} / D_{-}=1\right)$, the curve crosses the origin, while for unequal ion diffusion coefficients the curve is shifted horizontally in negative direction ( $\mathrm{KCl}$ electrolyte with $D_{+} / D_{-}=0.96$ and $\mathrm{NaCl}$ electrolytes with $D_{+} / D_{-}=0.65$ ) or in positive direction (KF electrolyte with $D_{+} / D_{-}=1.33$ ). 
(a)

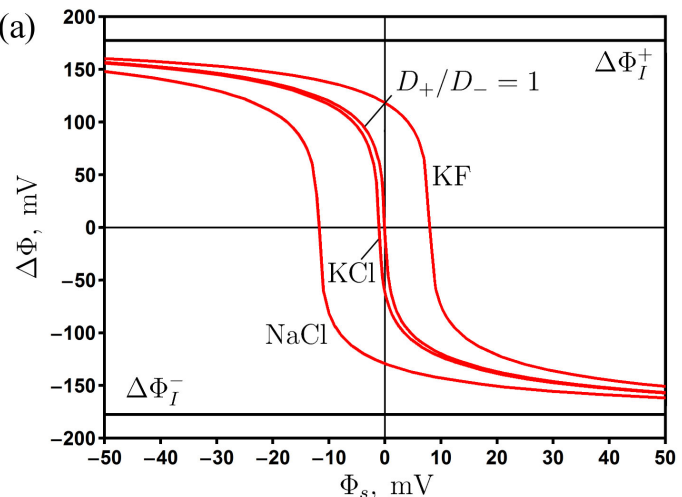

(b)

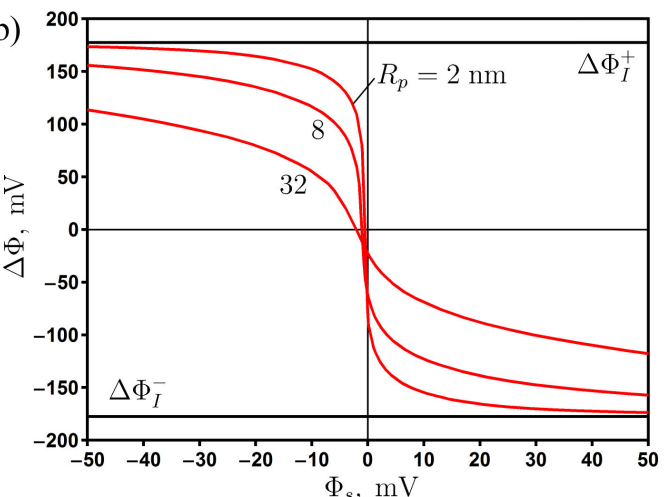

Fig. 4. The dependence of membrane potential on the potential applied to the pore surface for different electrolytes (a) and for different pore radii in $\mathrm{KCl}$ electrolyte (b). $C_{L}=100 \mathrm{mM}$, $C_{R}=0.1 \mathrm{mM}, R_{p}=8 \mathrm{~nm}, L_{p}=4000 \mathrm{~nm}$

The shift is caused by the diffusion potential, which is enhanced by the induced charge effect, see also Fig. 2 (b,d).

The effect of pore radius variation on the membrane potential is demonstrated in Fig. 4 (b). When the pore radius is small $\left(R_{p}=2 \mathrm{~nm}\right)$, the electric double layers from the opposite walls overlap. In this case, the membrane potential curve approaches the line of ideal cation (anion) selectivity when the applied potential is negative (positive). A sharp switch of selectivity is observed when $\Phi_{s}$ changes its sign. With increasing the pore radius, the ratio of Debye length to pore radius decreases, and the overlap of electric double layers becomes smaller and even vanishes at high concentration side $\left(C_{R}=100 \mathrm{mM}\right)$. The variation of membrane potential decreases indicating the decrease of ionic selectivity, while the transition from cation to anion selectivity becomes smooth when the applied potential changes its sign.

The obtained theoretical results on the membrane potential variation are qualitatively similar to the experimental data in $[7,8,10]$. The quantitative difference lies in the fact that in the experiment, a larger variation of applied potential (by an order of magnitude) is required to obtain the same change of membrane potential. The quantitative matching of experimental and theoretical data requires the extension of the present model by including the Stern layer, where a significant drop of potential could be observed. This problem will be addressed in future studies.

\section{Conclusion}

We have performed modelling of electrochemically switchable ion transport in nanoporous membrane with conductive surface on the basis of two-dimensional Space-charge model. The membrane separates two reservoirs with different salt concentrations. The impact of potential applied to the nanopore surface on the membrane potential at zero current is investigated. It is shown that the variation of applied potential from negative to positive values results in the continuous change of membrane selectivity from cation to anion. When ion diffusion coefficients are equal $\left(D_{+} / D_{-}=1\right)$, the dependence of membrane potential on the applied surface potential is an odd function at different fixed concentrations in the reservoirs. For unequal ion diffusion coefficients, the diffusion potential greatly enhanced by the induced charge effect leads to the 
shift of membrane potential curves along the applied potential axis to negative $\left(0<D_{+} / D_{-}<1\right)$ or positive $\left(D_{+} / D_{-}>1\right)$ direction. The decrease of pore radius results in the increase of ionic selectivity and steep transition between cation-selective and anion-selective states.

The reported study was funded by Russian Foundation for Basic Research, Government of Krasnoyarsk Territory, Krasnoyarsk Regional Fund of Science, to the research project 18 48-242011 "Mathematical modelling of synthesis and ionic transport properties of conductive nanoporous membranes".

\section{References}

[1] H.Strathmann, Ion-exchange membrane separation processes, Elsevier, Amsterdam, 2004.

[2] A.Figoli, J.Hoinkis, S.A.Altinkaya, J.Bundschuh, Application of nanotechnology in membranes for water treatment, CRC Press, 2017.

[3] F.G.Bănică, Chemical sensors and biosensors: fundamentals and applications, John Wiley \& Sons, Chichester, UK, 2012.

[4] M.Tagliazucchi, I.Szleifer, Transport mechanisms in nanopores and nanochannels: can we mimic nature? Mater. Today, 18(2015), no. 3, 131-142.

[5] Z.S.Siwy, S.Howorka, Engineered voltage-responsive nanopores, Chem. Soc. Rev., 39(2010), $1115-1132$.

[6] X.Hou, W.Guo, L.Jiang, Biomimetic smart nanopores and nanochannels, Chem. Soc. Rev., 40(2011), 2385-2401.

[7] M.Nishizawa, V.P.Menon, C.R.Martin, Metal nanotubule membranes with electrochemically switchable ion-transport selectivity, Science, 268(1995), 700-702.

[8] C.R.Martin, M.Nishizawa, K.Jirage, M.Kang, S.B.Lee, Controlling ion-transport selectivity in gold nanotubule membranes, Adv. Mater., 13(2001), 1351-1362.

[9] M.S.Kang, C.R.Martin, Investigations of potential-dependent fluxes of ionic permeates in gold nanotubule membranes prepared via the template method, Langmuir, 17(2001), 2753-2759.

[10] W.Guan, M.A.Reed, Electric field modulation of the membrane potential in solid-state ion channels, Nano Lett., 12(2012), 6441-6447.

[11] W.Guan, R.Fan, M.A.Reed, Field-effect reconfigurable nanofluidic ionic diodes, Nat. Commun., 2(2011), Article no. 506.

[12] P.Gao, C.R.Martin, Voltage charging enhances ionic conductivity in gold nanotube membranes, ACS Nano, 8(2014), 8266-8272.

[13] Q.Wang, C.S.Cha, J.Lu, L.Zhuang, Ionic conductivity of pure water in charged porous matrix, Chem. Phys. Chem., 13(2012), 514-519.

[14] D.V.Lebedev, A.V.Shiverskiy, M.M.Simunin, V.S.Solodovnichenko, V.A.Parfenov, V.V.Bykanova, S.V.Khartov, I.I.Ryzhkov, Preparation and ionic selectivity of carbon-coated alumina nanofiber membranes, Petrol. Chem., 57(2017), no. 4, 306-317. 
[15] V.S.Solodovnichenko, D.V.Lebedev, V.V.Bykanova, A.V.Shiverskiy, M.M.Simunin, V.A.Parfenov, I.I.Ryzhkov, Carbon coated alumina nanofiber membranes for selective ion transport, Adv. Engineer. Mater., 20(2017), 1700244.

[16] D.V.Lebedev, V.S.Solodovnichenko, M.M.Simunin, I.I.Ryzhkov. Effect of electric field on ion transport on nanoporous membranes with conductive surface, Petrol. Chem., 58(2018), no. $6,474-481$.

[17] I.I.Ryzhkov, D.V.Lebedev, V.S.Solodovnichenko, A.V.Shiverskiy, M.M.Simunin, Inducedcharge enhancement of the diffusion potential in membranes with polarizable nanopores, Phys. Rev. Lett., 119(2017), 226001.

[18] I.I.Ryzhkov, D.V.Lebedev, V.S.Solodovnichenko, A.V.Minakov, M.M.Simunin, On the origin of membrane potential in membranes with polarizable nanopores, J. Membr. Sci., 549(2018), 616-630.

[19] I.I.Ryzhkov, A.S.Vyatkin, A.V.Minakov. Theoretical study of electrolyte diffusion through polarizable nanopores, J. Siber. Fed. Univer.: Math. Phys., 11(2018), no. 4, 494-504.

[20] M.Z.Bazant, T.M.Squires, Induced-charge electrokinetic phenomena, Curr. Op. Coll. Inter. Sci., 15(2010), 203-213.

[21] S.Rubin, M.E.Suss, P.M.Biesheuvel, M.Bercovici, Induced-charge capacitive deionization: the electrokinetic response of a porous particle to an external electric field, Phys. Rev. Lett., $\mathbf{1 1 7}(2016), 234502$.

[22] C.Amatore, A.I.Oleinick, I.Svir, Theory of ion transport in electrochemically switchable nanoporous metallized membranes, Chem. Phys. Chem., 10(2009), 211-221.

[23] P.Ramírez, S.Mafé, A.Alcaraz, J.Cervera, Modeling of $\mathrm{pH}$-switchable ion transport and selectivity in nanopore membranes with fixed charges, J. Phys. Chem. B, 107(2003), $13178-13187$.

[24] R.B.Schoch, J.Han, P.Renaud, Transport phenomena in nanofluidics, Rev. Modern Phys., 80(2008), 839-883.

[25] R.Qiao, N.R.Aluru, Ion concentrations and velocity profiles in nanochannel electroosmotic flows, J. Chem. Phys., 118(2003), 4692-4701.

[26] V.E.Zalizniak, O.A.Zolotov, I.I. Ryzhkov, Effective molecular dynamics model of ionic solutions for large-scale calculations, J. Appl. Mech. Tech. Phys., 59(2018) 41-51.

[27] K.L.Yang, S.Yiacoumi, C.Tsouris, Monte Carlo simulations of electrical double-layer formation in nanopores, J. Chem. Phys., 117(2002), 8499-8507.

[28] H.Yoshida, H.Mizuno, T.Kinjo, H.Washizu, J.L.Barrat, Molecular dynamics simulation of electrokinetic flow of an aqueous electrolyte solution in nanochannels, J. Chem. Phys., 140(2014), 214701.

[29] D.Duong-Hong, J.S.Wang, G.R.Liu, Y.Z.Chen, J.Han, N.G.Hadjiconstantinou, Dissipative particle dynamics simulations of electroosmotic flow in nano-fluidic devices, Microfluid Nanofluid, 4(2008), 219-225. 
[30] R.J.Gross, J.F.Osterle, Membrane transport characteristics of ultrafine capillaries, J. Chem. Phys., 49(1968), 228-234.

[31] P.B.Peters, R.van Roij, M.Z.Bazant, P.M.Biesheuvel, Analysis of electrolyte transport through charged nanopores, Phys. Rev. E, 93(2016), 053108.

[32] S.Levine, J.R.Marriott, G.Neale, N.Epstein, Theory of electrokinetic flow in fine cylindrical capillaries at high zeta-potentials, J. Coll. Inter. Sci., 52(1975), no. 1, 136-149.

\title{
Моделирование управляемого транспорта ионов через нанопористые мембраны с проводящей поверхностью
}

\author{
Илья И. Рыжков \\ Антон С. Вяткин \\ Институт вычислительного моделирования СО РАН \\ Академгородок, 50/44, Красноярск, 660036 \\ Сибирский федеральный университет \\ Свободный, 79, Красноярск, 660041 \\ Россия \\ Мария И. Медведева \\ Сибирский федеральный университет \\ Свободный, 79, Красноярск, 660041 \\ Россия
}

\begin{abstract}
В работе теоретически исследовано влияние потенииала, приложенного к проводящей поверхности нанопористой мембраны, на мембранный потенциал при нулевом токе на основе двумерной модели пространственного заряда. Мембрана разделяет два резервуара с различными концентрациями соли. Показано, что изменение приложенного потенциала в направлении от отрицательных значений $к$ положительным приводит $к$ непрерывному изменению селективности мембраны от катиона к аниону. При одинаковых коэффициентах диффузии ионов зависимость мембранного потенциала от приложенного является нечетной функцией, в то время как при различных коэффициентах дифбузии эта зависимость сдвигается вдоль оси приложенного потенциала за счет вклада диффузионного потенииала, усиленного присутствием индуцированного заряда на проводящей поверхности. Уменъшение радиуса поры приводит к увеличению ионной селективности и более резкому переходу от катион- $к$ анион-селективному состоянию при изменении приложенного потенциала.
\end{abstract}

Ключевые слова: нанопористая мембрана, двойной электрический слой, перенос электролита, мембранный потенциал, модель пространственного заряда. 